\title{
Pacific
}

Journal of

Mathematics

\section{VOLUMES OF TUBULAR NEIGHBOURHOODS OF REAL}

\section{ALGEBRAIC VARIETIES}

\author{
RICHARD ALEXANDER WONGKEW
}




\title{
VOLUMES OF TUBULAR NEIGHBOURHOODS OF REAL ALGEBRAIC VARIETIES
}

\author{
RICHARD WONGKEW
}

This paper concerns the following problem:

Let $V$ be an algebraic variety in $n$-dimensional euclidean space. For each pair of positive numbers $\rho$ and $R$ find an upper bound on the volume of the set of points that are within a distance of $\rho$ from $V$ and within a distance $R$ from a fixed point $p_{0}$. Obtain this upper bound so that it is independent of the choice of $p_{0}$. In particular, does there exist a universal $n$ th-degree polynomial, say $P_{n}(\cdot, \cdot, \cdot)$ which automatically provides an upper bound upon entering $\rho, R$ and the degree of $V$ ?

Introduction. Among the people who have worked on this problem are Demmel, Renegar, Ocneanu and myself. The one common factor which led us to this problem was our familiarity with Smale's Bulletin article [S]. In it Smale obtained an upper bound for the case where $V$ is a complex hypersurface. His method however lacks rigor and possesses a serious flaw. This flaw involves incorrectly applying Fubini's Theorem and ignoring the special points of $V$ where the "relative curvature" is larger than $\Omega\left(\frac{1}{\rho}\right)$. Since then Renegar who was a thesis student of Smale has obtained a correct solution to this special case where $V$ is a complex algebraic hypersurface. Later on, Demmel using some elementary results from integral geometry extended Renegar's results to all complex algebraic varieties and all tubes of length less than 1. See Theorem 4.1 of Demmel [D]. Crucial to Demmel's result is an estimate of the $r$-dimensional measure of the portion of an $r$-dimensional algebraic variety contained in an $n$-ball $S$ with radius $R$. More specifically Demmel used the following fact: There exists a constant $c$ depending only on $n$, so that if $\operatorname{Vol}_{r}(\cdot)$ is the $r$-dimensional measure of $r$-manifolds, then

$$
\operatorname{Vol}_{r}(V \cap S) \leq c \cdot \operatorname{degree}(V) \cdot R^{r}
$$

For the methods used in proving this result one can see Wongkew ([W], pp. 9-10) or Demmel ([D], p.19). This fact generalizes Proposition 6.3 in Renegar [R]. 
Independently of Renegar, Adrian Ocneanu obtained the most general result of all. Unfortunately, Ocneanu did not publish his result and only a handwritten rough draft is in circulation. Ocneanu gave a talk on his result and the methods he used in achieving it. See [O]. In this talk he gave the following estimate:

$$
\operatorname{Vol}_{n}\left[T_{\rho}(V \cap S)\right] \leq \sum_{k=m}^{n} 2 m O_{n} C_{k}^{n}(d \rho)^{k} R^{n-k}
$$

Here $C_{k}^{n}$ is the number of ways to choose $k$ things from $n, d$ is the algebraic degree of $V, m$ is the codimension of $V$ and $O_{n}$ is the volume of the unit $n$-ball. Crucial to Adrian's proof of this inequality was his ability to express the infinitesimal volume form of a manifold in terms of a sum of symmetric functions of its principal curvatures. Looking through the standard references on geometry and measure theory I have only been able to find such formulas for convex bodies in $E^{n}$.

1. Using purely topological and algebraic arguments I will demonstrate the existence of a solution to our problem. More precisely, I will prove the following.

MAIN THEOREM. Let $m$ be the codimension of a real algebraic variety $V$ whose defining polynomials are all bounded in degree by $d$. And let $S$ be an arbitrary $n$-ball in $E^{n}$ with radius $R$. There exist constants $\left\{c_{j}\right\}$ which depend only on $n$, so that for all positive $\rho$ the following is true:

$$
\operatorname{Vol}_{n}\left[T_{\rho}(V \cap S)\right] \leq \sum_{j=m}^{n} c_{j} d^{j} \rho^{j} R^{n-j} .
$$

A variation of this theorem can be derived from a result due to Weyl [We]. However, in this variation, the elements of $\left\{c_{j}\right\}$ depend on integral invariants of $V \cap S$ as well as the degree of $V$; furthermore, the size of $\rho$ is bounded.

In this paper we will use $|\cdot|$ to signify either the length (norm) of a vector (operator) or the number of elements in a set. The meaning will be clear from the context.

Proof of Main Theorem. From this point onwards the symbols $\rho$ and $R$ will represent non-negative real numbers and $V$ will represent a real algebraic variety of degree $d$ and codimension $m$ in $E^{n}$. Let $(\rho, R, V)$ be a randomly chosen triple. Assume that $S$ is a randomly 
chosen $n$-ball with radius $R$. We will apply induction on $n$. So assume $n=1$. The Fundamental Theorem of Algebra tells us that $V$ is a finite set with $|V| \leq d$. Thus for $n=1$ we have

$$
\operatorname{Vol}_{n}\left[T_{\rho}(V \cap S)\right] \leq 2^{n} \cdot(d \rho)^{n} .
$$

Thus our main theorem is obviously true for $n=1$. So assume our theorem is true for algebraic varieties that are defined by $n-1$ or less variables. To carry out our inductive argument, we will need some results from Differential Topology and Algebraic Geometry. More specifically we will need the following:

Theorem (M). ${ }^{1}$ Let $V$ be a real algebraic variety in $n$-dimensional euclidean space. If each of the defining polynomial $f$ for $V$ has degree bounded above by $d$, then the sum of the Betti numbers of $V$ is bounded above by the following nth degree polynomial in $d$ :

$$
O(d)=d(2 d-1)^{n-1} \text {. }
$$

TheOREM (S). ${ }^{2}$ For any topological space $\mathbf{X}$, the 0th Homology group for $\mathbf{X}$ is a free group. The rank of this free group equals the number of non-empty components of $\mathbf{X}$.

By a change of coordinates of $E^{n}$ we may assume that $S$ is centered at the origin. Thus $S$ is the standard $R$-ball, $\{|x| \leq R\}$. And it is clear that for any pair of positive numbers $\rho$ and $R$, we can cover the ball $S$ with regular cubes ${ }^{3}\left\{V_{k}\right\}$ which satisfy the following five properties:

(1) There exists a family of hyperplanes $G_{\rho}^{R}=\left\{H_{\alpha}\right\}$ so that each $V_{k}$ is in the closure of a component of $S \sim \cup H_{\alpha}$; and each hyperplane $H_{\alpha}$ is normal to $e_{j}$ for some $j$.

(2) $V_{k} \cap V_{j} \neq \varnothing$ implies $k=j$ or $V_{k} \cap V_{j}$ is a regular $n-1$ dimensional cube in $R^{n}$.

(3) The diameter of each $V_{k}$ is less than $\rho \sqrt{n} / n$.

(4) If $H \in G_{\rho}^{R}$ then $V \cap H$ is a real algebraic variety which satisfies the following:

(a) dimension of $V \cap H=n-m-1=\operatorname{dim}(V)-1$.

(b) the algebraic degree ${ }^{4}$ of $V \cap H=d=$ degree of $V$.

\footnotetext{
${ }^{1}$ See Theorem 2 of Milnor [M], p. 275.

${ }^{2}$ For Theorem [S] see Corollary 8 of Spanier [Sp], p. 175.

${ }^{3}$ Here I am using the term regular cube to refer to an $n$-dimensional rectangle; i.e. all the edges are perpendicular.

${ }^{4}$ By the algebraic degree of a variety we mean the degree of the defining polynomials for that variety.
} 
(5) For each $j$, the number of hyperplanes in $G_{\rho}^{R}$ with normal vector $e_{j}$ is bounded above by $2 n R / \rho$.

Assume $\left\{V_{k}\right\}$ and $G_{\rho}^{R}$ are cubes and hyperplanes which satisfy (1) through (5) above; then the following inequalities are true:

$$
\begin{aligned}
\operatorname{Vol}_{n}\left[T_{\rho}(V \cap S)\right] \leq & \left\{\sum_{H \in G_{\rho}^{R}} \operatorname{Vol}_{n}\left[T_{\rho+\rho / n}(V \cap H \cap S)\right]\right\} \\
& +E(\rho, V, R) .
\end{aligned}
$$

Here $E(\rho, V, R)$ is equal to the volume of $T_{\rho}\left(S_{\rho}^{R}\right)$ and $S_{\rho}^{R}$ is defined thus:

Definition. For each $x \in V$ let $h(x)$ be the distance from $x$ to $\cup\left[V \cap H: H \in G_{\rho}^{R}\right]$. Then $S_{\rho}^{R}$ is the set of points $x \in V \cap S$ where $h(x) \geq \rho / \sqrt{n}$.

From our induction hypothesis, there exist $n-1$ constants $\left\{c_{j}\right\}$, so that for all hyperplanes $H \in G_{\rho}^{R}$ we have

$$
\operatorname{Vol}_{n-1}\left[T_{\rho}(V \cap H \cap S)\right] \leq \sum_{m}^{n-1} c_{j} d^{j} \rho^{j} R^{n-1-j}
$$

This implies that

$$
\operatorname{Vol}_{n}\left[T_{\rho}(V \cap H \cap S)\right] \leq \sum_{m}^{n-1} c_{j} d^{j} \rho^{j+1} R^{n-(1+j)} .
$$

Now for each $i, 1 \leq i \leq n$, let $G_{i}$ be the set of all hyperplanes in $G_{\rho}^{R}$ with normal vector $e_{i}$. From properties (5) and (1) we have for each $i$,

$$
\left|G_{i}\right| \leq \frac{2 n R}{\rho} \text { and } G_{\rho}^{R}=\bigcup\left(G_{i}: 1 \leq i \leq n\right) .
$$

This plus the inequalities above imply that for each $i$,

$$
\begin{aligned}
\sum_{H \in G_{i}} \operatorname{Vol}_{n}\left[T_{\rho}(V \cap H \cap S)\right] & \leq \sum_{j=m}^{n-1} \frac{2 n R}{\rho} c_{j} d^{j} \rho^{j+1} R^{n-(j+1)} \\
& =\sum_{j=m}^{n-1} 2 n c_{j} d^{j} \rho^{j} R^{n-j}
\end{aligned}
$$

Since $i$ only takes values between 1 and $n$, we know that for some $i$., the following is true:

$$
\sum_{H \in G_{\rho}^{R}} \operatorname{Vol}_{n}\left[T_{\rho}(V \cap H \cap S)\right] \leq n \sum_{H \in G_{i_{\bullet}}} \operatorname{Vol}_{n}\left[T_{\rho}(V \cap H \cap S)\right]
$$


Combining this with (4) we get

$$
\sum_{H \in G_{\rho}^{R}} \operatorname{Vol}_{n}\left[T_{\rho}(V \cap H \cap S)\right] \leq \sum_{j=m}^{n-1} 2 n^{2} c_{j} d^{j} \rho^{j} R^{n-j} .
$$

Thus upon replacing $\rho$ with $\rho+\rho / n$ in (6) and plugging the result into (1) we get

$$
\begin{aligned}
\operatorname{Vol}_{n}\left[T_{\rho}(V \cap S)\right] \leq & \sum_{j=m}^{n-1} 2 n^{2} c_{j}\left(1+\frac{1}{n}\right)^{j} d^{j} \rho^{j} R^{n-j} \\
& +\operatorname{Vol}_{n}\left[T_{\rho}\left(V \cap S_{\rho}^{R}\right)\right] .
\end{aligned}
$$

Now for each $1 \leq j \leq n-1$, let us redefine $c_{j}$ to be equal to $2 n^{2} c_{j}\left(\frac{1+n}{n}\right)^{j}$. Then we can rewrite (7) as

$$
\operatorname{Vol}_{n}\left[T_{\rho}(V \cap S)\right] \leq \sum_{j=m}^{n-1} c_{j} d^{j} \rho^{j} R^{n-j}+\operatorname{Vol}_{n}\left[T_{\rho}\left(V \cap S_{\rho}^{R}\right)\right]
$$

Clearly, we are finished as soon as we demonstrate the existence of a constant $c_{n}$ independent of $\rho, R$, and $V$ so that

$$
\operatorname{Vol}_{n}\left[T_{\rho}\left(V \cap S_{\rho}^{R}\right)\right] \leq c_{n}(d \rho)^{n} .
$$

Our proof of (9) follows from the following:

Claim. If $O_{n}$ is the volume of the unit ball in $E^{n}$ and we set $c_{n}=3^{n} O_{n}$ then (9) is true for all $R, \rho, V$ and $d$.

To prove our claim we will need the following lemma:

LEMMA (C). Let $d$ be the degree of the defining polynomials for an algebraic variety $V$ in $n$-dimensional euclidean space. Then the number of components of $V$ is bounded above by

$$
d(2 d-1)^{n-1} \text {. }
$$

Proof. By definition the 0th Betti number of $V$ is the rank of the 0th Homology group of $V$. And from Theorem (S) we know that the number of connected components of $V$ is equal to the 0th Betti number of $V$. Thus in a rather crude fashion, it follows that the sum of the Betti numbers $V$ is an upper bound for the number of bounded components of $V$. From the works of Milnor [M], Thom [T] and Oleinik [Ol] we know this sum of Betti numbers is bounded 
above by an $n$th degree polynomial in $d$. From Theorem (M) we may assume this polynomial is

$$
d(2 d-1)^{n-1} .
$$

Thus we have completed the proof of our lemma.

Proof of Claim. Let $\left\{U_{k}\right\}$ be the set of components of $E^{n} \sim$ $\bigcup\left\{H \in G_{\rho}^{R}\right\}$ which contains $S_{\rho}^{R}$. Now $\left\{U_{k}\right\}$ is defined as a cover of $S_{\rho}^{R}$; and the elements of $S_{\rho}^{R}$ are bounded components of $V$. From Lemma (C), the number of components of $V$ is bounded above by

$$
O(d)=d(2 d-1)^{n-1} .
$$

Thus the following inequality is true:

$$
\left|\left\{U_{k}\right\}\right|=\text { cardinality of }\left\{U_{k}\right\} \leq d(2 d-1)^{n-1} .
$$

But because $\left\{U_{k}\right\}$ covers $S_{\rho}^{R}$ we get

$$
T_{\rho}\left(S_{\rho}^{R}\right) \subset T_{\rho}\left[\bigcup U_{k}\right]
$$

Thus

$$
\operatorname{Vol}_{n} T_{\rho}\left(S_{\rho}^{R}\right) \leq \operatorname{Vol}_{n} T_{\rho}\left[\bigcup U_{k}\right] .
$$

From the sub-additivity of Lebesgue measure we know that

$$
\operatorname{Vol}_{n} T_{\rho}\left[\bigcup U_{k}\right] \leq \sum_{k} \operatorname{Vol}_{n} T_{\rho}\left[U_{k}\right]
$$

Now each $U_{k}$ is the result of a translation of the $n$-cube, $\left[0, \frac{\rho}{n}\right]^{n}$. Thus upon combining this fact with (10) and (12) we get

$$
\mathrm{Vol}_{n} T_{\rho}\left[\bigcup U_{k}\right] \leq d(2 d-1)^{n-1} \operatorname{Vol}_{n}\left[T_{\rho}\left(\left[0, \frac{\rho}{n}\right]^{n}\right)\right] .
$$

Now the center of the $n$-cube, $\left[0, \frac{\rho}{n}\right]^{n}$ is the point $p_{0}=\frac{\rho}{2 n} \sum_{k=1}^{n} e_{k}$. And every point of the $n$-cube, $\left[0, \frac{\rho}{n}\right]^{n}$ is within a distance of $\frac{\rho}{2}$ from $p_{0}$. In other words, the following relation holds:

$$
\left[T_{\rho}\left(\left[0, \frac{\rho}{n}\right]^{n}\right)\right] \subset\left(x:\left|x-p_{0}\right| \leq \frac{3 \rho}{2}\right) .
$$

This implies that for each $U_{k}$,

$$
\operatorname{Vol}_{n}\left[T_{\rho}\left(U_{k}\right)\right]<\operatorname{Vol}_{n}\left[\left|p-p_{0}\right| \leq \frac{3 \rho}{2}\right]=O_{n} \cdot\left(\frac{3}{2} \rho\right)^{n} \text {. }
$$


Upon combining (11) and (13) with our previous inequality we obtain that

$$
\operatorname{Vol}_{n} T_{\rho}\left(S_{\rho}^{R}\right)<(d)(2 d-1)^{n-1} \cdot O_{n}\left(\frac{3}{2} \rho\right)^{n}
$$

From this inequality and the fact that

$$
(d)(2 d-1)^{n-1} \cdot O_{n}\left(\frac{3}{2} \rho\right)^{n}<3^{n} O_{n}(d \rho)^{n}
$$

we see that we have proven our claim; and thus the proof of our Main Theorem is complete.

Postscript. It is a little unsettling to use the sum of the Betti numbers as the upper bound on the number of components. It is only the 0th Betti number of $V$ that one needs for the proof. One would hope that we could get a much sharper bound for this. Thus I shall end with some of the questions which bothered me throughout the paper:

1. Are there sharp estimates for the 0th Betti number of $V$ in terms of $d$ and $n$ ?

2. What are the best constants for the formula in our main theorem?

3. Is there a formula for expressing the infinitesimal volume form of an arbitrary singular (non-singular) submanifold in terms of a sum of symmetric functions of its principal curvatures?

\section{REFERENCES}

[A] L. Auslander and R. E. Mackenzie, Introduction to Differentiable Manifolds, McGraw-Hill Series in Higher Math., 1963.

[BT] R. Bott and L. Tu, Differential Forms in Algebraic Topology, Springer-Verlag, New York, 1982.

[D] J. Demmel, The probability that a numerical analysis problem is difficult, Courant Institute, New York University, preprint 1987.

[DFN] B. A. Dubrovin, A. T. Fomenko and S. P. Novikov, Modern GeometryMethods and Applications. Part II. The Geometry and Topology of Manifolds, Springer-Verlag, New York, 1985.

[H] H. Hotelling, Tubes and spheres in n-spaces, and a class of statistical problems, Amer. J. Math., 61 (1939), 440-446.

[HY] J. Hocking and G. Young, Topology, Addison-Wesley, Reading, Mass., 1961.

[K] E. Kostlan, Complexity Theory of Numerical Linear Algebra, preprint M.S.R.I. 1985.

[M] J. Milnor On the Betti numbers of real varieties, Proc. Amer. Math. Soc., 15 (1964), 275-280.

[O] A. Ocneanu, On the Volumes of Tubular Neighbourhoods of Algebraic Varieties, presentation 1984, MSRI, Berkeley. 
[O1] O. A. Oleinik, Estimates of the Betti numbers of real algebraic hypersurfaces, Mat. Sb. (N.S.), 28 (70) (1951), 635-640 (Russian).

[R] J. Renegar, On the Efficiency of Newton's Method in Approximating all Zeros of a System of Complex Polynomials, preprint M.S.R.I. 1985.

[S] S. Smale, The fundamental theorem of algebra and complexity theory, Bull. Amer. Math. Soc. (N.S.), 4 (1981), 1-36.

[Sp] E. H. Spanier, Algebraic Topology, McGraw-Hill Series in Higher Mathematics, 1966.

[T] R. Thom, Sur l'Homologie des Variétés Algébriques Relles in A Symposium in Honor of Marston Morse, Princeton University Press, Stuart S. Cairnes (editor) 1965.

[Wa] B. L. Van Der Waerden, Modern Algebra, vol. 2, Ungar, New York 1950.

[We] H. Weyl, On the volume of tubes, Amer. J. Math., 61 (1939), 461-472.

[Wo] R. Wongkew, Partitioning for Strong Convergence of Newton's Method, Mathematics Dept. UC Berkeley, preprint 1985.

Received December 9, 1989 and in revised form November 25, 1991.

UNIVERSITY OF THE VIRGIN ISLANDS

ST. Thomas, VI 00802

AND

WORLD INSTITUTE FOR SCIENCE

KINGSTON

JAMAICA, WeST INDIES 


\section{PACIFIC JOURNAL OF MATHEMATICS}

Founded by

E. F. BECKENBACH (1906-1982) F. Wolf (1904-1989)

\section{EDITORS}

\author{
V. S. VARADARAJAN \\ (Managing Editor) \\ University of California \\ Los Angeles, CA 90024-1555 \\ vsv@math.ucla.edu \\ F. Michael Christ \\ University of California \\ Los Angeles, CA 90024-1555 \\ christ@math.ucla.edu
}

\section{Herbert Clemens}

University of Utah

Salt Lake City, UT 84112

clemens@math.utah.edu

\author{
ThOMAs ENRIGHT \\ University of California, San Diego \\ La Jolla, CA 92093 \\ tenright@ucsd.edu \\ Nicholas Ercolani \\ University of Arizona \\ Tucson, AZ 85721 \\ ercolani@math.arizona.edu \\ R. FINN \\ Stanford University \\ Stanford, CA 94305 \\ finn@gauss.stanford.edu \\ VAUGHAN F. R. Jones \\ University of California \\ Berkeley, CA 94720 \\ vfr@math.berkeley.edu
}

STEVEN KeRCKHOFF

Stanford University

Stanford, CA 94305

spk@gauss.stanford.edu

Martin ScharlemanN

University of California

Santa Barbara, CA 93106

mgscharl@henri.ucsb.edu

Harold Stark

University of California, San Diego

La Jolla, CA 92093

\section{SUPPORTING INSTITUTIONS}

UNIVERSITY OF ARIZONA

UNIVERSITY OF BRITISH COLUMBIA

CALIFORNIA INSTITUTE OF TECHNOLOGY

UNIVERSITY OF CALIFORNIA

UNIVERSITY OF MONTANA

UNIVERSITY OF NEVADA, RENO

NEW MEXICO STATE UNIVERSITY

OREGON STATE UNIVERSITY
UNIVERSITY OF OREGON

UNIVERSITY OF SOUTHERN CALIFORNIA

STANFORD UNIVERSITY

UNIVERSITY OF HAWAII

UNIVERSITY OF UTAH

WASHINGTON STATE UNIVERSITY

UNIVERSITY OF WASHINGTON 


\section{PACIFIC JOURNAL OF MATHEMATICS}

Volume 159 No. $1 \quad$ May 1993

An application of the very weak Bernoulli condition for amenable groups

SCOT ROBERT ADAMS and JEFFREY EDWARD STEIF

An application of homogenization theory to harmonic analysis on solvable Lie groups of polynomial growth

G. Alexopoulos

The standard double soap bubble in $\mathbf{R}^{2}$ uniquely minimizes perimeter

Joel Foisy, Manuel Alfaro Garcia, JefFrey FARlowe

Brock, NiCKELOUS Hodges and JASON ZimbA

Pseudo regular elements and the auxiliary multiplication they induce

BARRY E. JOHNSON

A converse to a theorem of Komlós for convex subsets of $L_{1}$

CHRISTOPHER JOHN LENNARD

General Kac-Moody algebras and the Kazhdan-Lusztig conjecture

WAYNE L. NEIDHARDT

The flow space of a directed $G$-graph

WILLIAM LINDALL PASCHKE

Primitive ideals and derivations on noncommutative Banach algebras

MARK PHILLIP THOMAS

Equivariant Nielsen numbers

PETER N-S WONG

Volumes of tubular neighbourhoods of real algebraic varieties

RichaRd ALEXANDER WONGKEW

The intrinsic group of Majid's bicrossproduct Kac algebra 